\title{
Emission of High Energy during Super-Compressibility of Supersonic Jets
}

\author{
Kholmurad Khasanov \\ Gas and Wave Dynamics Department, M. V. Lomonosov Moscow State University, \\ Moscow, Russia \\ Email:kholkh@bk.ru
}

Received August 31, 2012; revised October 3, 2012; accepted October 15, 2012

\begin{abstract}
New source of light emission of high energy is found due to arising of instability in supersonic jets. These phenomena are observed in gas jets flowing from the nozzle with a central cone. It leads to high accelerations of the molecules, ions and elementary particles. The emission spectra of the jets are obtained. Decoding of the spectra allowed us to define inverted population of rotational and vibrational levels, electrons temperature, rotational and vibrational temperatures for molecular ions. Internal energy decreasing provides the instability and gas volume decreasing due to internal forces; super-compressibility is result of it; its produce high density of light energy emission in various continuous media.
\end{abstract}

Keywords: Internal Energy Decreasing; Light of High Density of Electromagnetic Energy Emission; Super-Compressibility; Supersonic Jets

\section{Introduction}

The emission of electromagnetic radiation of high energy during super-compressibility of supersonic spiral-twisted jets is observed. High energy density is detected in gas flowing from the nozzle with a central cone [1-4]. This nozzle is acoustic resonator and dynamic emitter [5]. The phenomenon of super-compressibility in under-expanded submerged jet on output of the dynamic emitter results to the arising of rigid wave structures in gas media. These structures form the stable rigid boundary layers and emit high energy radiation, as summarized in $[6,7]$. The results of measurement of the characteristics of these fields are presented in this work.

Super-compressibility of the jet that accompanied by high energy radiation is close to multibubble compressibility at sonoluminescence phenomenon in liquid [8]. But the phenomena discussed in present paper have significant differences from sonoluminescence and were found in the gas flows. The evaluation of linear instability of the waves as sources of sound in a supersonic turbulent jet was discussed in [9]. They show that the noise generation process is not well modeled quantitatively by the linear theory. Their theoretical approach may be useful to explain our experiments.

Many open questions remain in the study of structured jets. Similar phenomena are observed during the jets emanating from young stellar objects. These non-relativistic beams of supersonic plasma are known to cool effectively via radiation losses. Of particular interest for astrophysics are issues related to the internal energy and internal structure of the jet [10].

Recent observations using Hubble Space Telescope and other high-resolution platforms indicate that jets may content significant sub-radial structure $\left(\delta x<r_{j e t}\right)$ with high Mach numbers $(M \sim 20)$, which implies that jets may be inherently heterogeneous. Thus experimental studies to date have shown that stable supersonic jets can propagate over long distances without energetic losses and without disruption of the structure [11].

The jets studied are both lighter and heavier than the ambient and meet the two main scaling parameter requirements for proto-stellar jets: the ejection Mach number and the (ambient/jet) density ratio [12].

In presented experiments air and vapor-air jets form channels of rigid boundary layers with progressing strong compression of jet transverse and longitudinal crosssections. The super-compressibility we are discussing is result of the multiple adiabatic expansion of the gas in dynamic emitter that results to its internal energy decreasing and kinetic energy increasing.

The lifetime of the gas molecules in pre-chamber of the dynamic emitter defines the minimal intermolecular distance. The interaction leads to its high accelerations in the vicinity of the outlet of the annular nozzle. Heavy free ions, as $\left(\mathrm{OH}^{-}\right)$and $\left(\mathrm{N}_{2}^{+}\right)$, originating in the channels acquire relativistic accelerations.

In $[6,7]$ was estimated that force of attraction between 
molecules of oxygen is $18.9 \times 10^{-11} \mathrm{~N}$; the acceleration of oxygen molecule is $3.57 \times 10^{14} \mathrm{~m} / \mathrm{sec}^{2}$; the acceleration for hydrogen molecule is $1.2 \times 10^{16} \mathrm{~m} / \mathrm{sec}^{2}$. Such accelerations are the cause of gas super-compressibility and collapse of its wave structure.

The high energy radiation is a consequence of the formation of cold non-equilibrium plasma in the structured flows. The radiation had as regular and irregular character.

By the other way instead the jets with high Mach numbers $(M \sim 20)$ in present work we provide propagation of structured jets without disruption over long distances for low $(M \sim 1-3)$ Mach numbers and also for subsonic jets. Their propagation is accompanied with high energy radiation and cool effectively due to gas internal energy decrease and via radiation losses. We have attempted to develop the devices that can create steady jet beams as a starting point for further work.

\section{Experimental Part}

\subsection{Description of Gas Dynamic Part}

The present paper describes a supersonic jet of unusual configuration, which escapes into the atmosphere from a special annular nozzle with a conic central body. The design of the nozzle is shown in the Figure 1. In our experiments the length of the wave structure was equal to 10 diameters of outlet of the nozzle approximately. This unexpected phenomenon of a formation of self-constrictive spiral twisted configuration may have various practical applications and it can lead to new tasks of a development of gas-dynamical theory for his theoretical understanding in future. The jet with such spiral twisted configuration presents a new type of vortical flows.

The nozzle was made from bronze and other metals. In case of water vapor jets nozzle was made from composite material or meta-material as textolite.

Design of the nozzle in Figure 1 allows a longitudinal movement of the conic central body 1 inside the truncated cone $\mathbf{2}$ for a different disposition of its top relative to a cut of the nozzle. We have provided gas-dynamical and energetic researches of other configurations of under-expanded submerged jets during their flowing from this nozzle at different operating conditions and dimensional parameters. The phenomenon takes place under conditions (1) and (2):

$$
\begin{gathered}
a \cdot \operatorname{tg} \beta \leq b \cdot \operatorname{tg} \alpha ; \\
\frac{\pi}{30}<\alpha<\frac{\pi}{2} .
\end{gathered}
$$

The influence of angles $\alpha$ and $\beta$ on the character of jets was studied in the ranges (3)-(4):

$$
39^{\circ} \leq \alpha \leq 132^{\circ}
$$

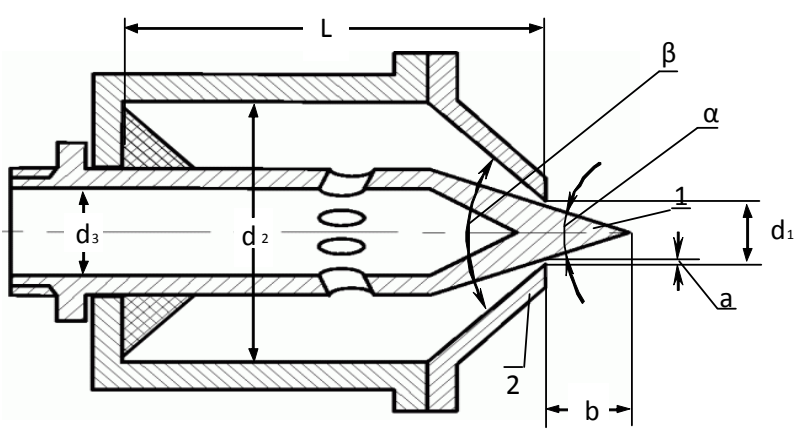

Figure 1. Design of the dynamic emitter with central cone. Here: 1-the central cone with diameter $d_{3}=25 \mathrm{~mm}$ and the angle $\alpha=30^{\circ}, 2$ - the truncated cone of the pre-chamber with diameter $d_{2}=60 \mathrm{~mm}$ and the angle $\beta=60^{\circ}, d_{1}$ is a diameter of outlet of the nozzle; the clearance of the controlled critical cut of the slit $a=0.3-0.6 \mathrm{~mm}$, the external part o the internal cone $b=3-6 \mathrm{~mm}$, a length of pre-chamber $L=150 \mathrm{~mm}$.

$$
\beta-\alpha \geq 12^{\circ} \text {. }
$$

Relations (1)-(4) provide the optimal conditions for emergence of regular interacting subsonic and supersonic jets. We altered gas pressure and geometry of prechamber. Compressor provided filtered gas pressure equal to 1.5 - $31 \mathrm{~atm}$.

When the gas is flowing out into the atmosphere it acquires a configuration of strong pronounced compressed flow of spiral-twisted radiating wave structures. The regular axial symmetric vortex structures in water vapor and air are obtained. These structures consist of the cells of certain dimensions. The type of structure and dimensions of its cells depends on the position of the central cone and pre-chamber pressure (see Figure 1).

In the Figure 2 there are presented shadowgraphs during different disposition of cone top relative to a cut of the nozzle. One can see that linear dimensions of the cells of jet structure are decreased during get out of the cone from outlet of the nozzle. All numbers are in $\mathrm{mm}$. The decreasing of indicated transverse and longitudinal dimensions of the jet demonstrates phenomenon of super-compressibility of the gas wave structure.

The super-compression of cell dimensions in Figure 2 occurs instantly (no more than $20 \mathrm{~ns}$ ) as result of super-compressibility of the jet and of its structure. The linear dimensions of jet structure depend on the position of central cone. As it seen from Figure 2, jet diameter 6 $\mathrm{mm}$ has became $5 \mathrm{~mm}$, distance between the nearest nodes of jet structure $10 \mathrm{~mm}$ has became $8.5 \mathrm{~mm}$ and jet diameter $4 \mathrm{~mm}$ has became $3 \mathrm{~mm}$ after 13 calibers of the nozzle.

The caliber is $\mathbf{d}_{\mathbf{1}}$ from Figure 1, or diameter of outlet of the annual nozzle. The jets presented in Figures $\mathbf{2}$ and $\mathbf{3}$ are stable supersonic jets.

Supersonic spiral-twisted air jets during their flowing 


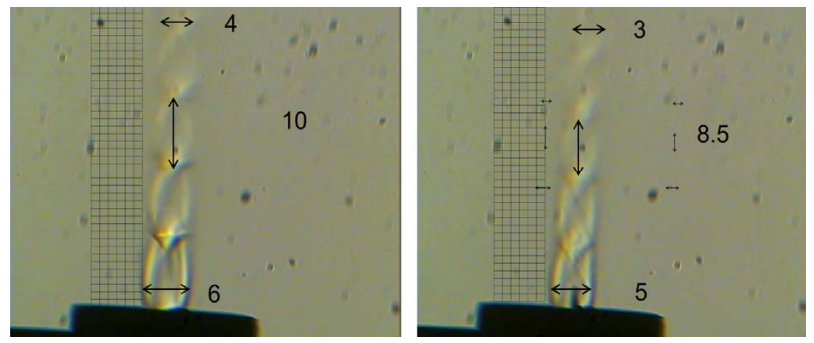

Figure 2. The shadowgraphs show two cases of gas flowing from the nozzle with a central cone during different disposition of cone top relative to a cut of the nozzle. Here on the left central cone was on $2.5 \mathrm{~mm}$ inside the outlet of nozzle and on the right it was on $\mathbf{2 . 5} \mathbf{~ m m}$ outside the outlet of nozzle. Pre-chamber pressure is $6 \mathrm{~atm}$ in both cases.
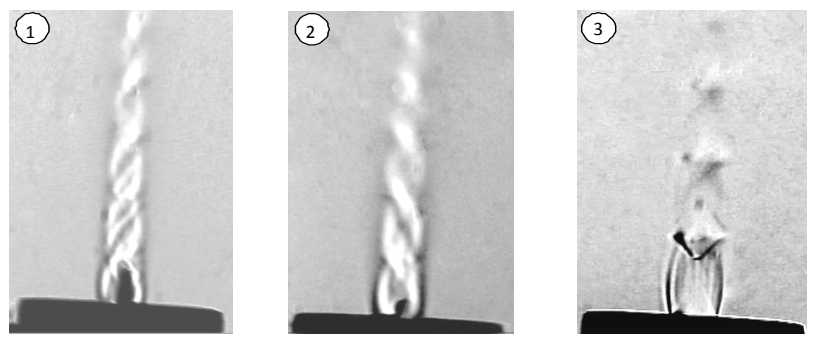

Figure 3. The shadowgraphs of instabilities of supersonic jets that lead to formation of right-(1) and left-twisted (2) rigid boundary layers as "horn of the unicorn". The direction of twist of spiral air jet is changing versus different positions of the central cone. A pressure in pre-chamber is equal $6 \mathrm{~atm}$ and a temperature of surrounding air is equal to $291^{\circ} \mathrm{K}$. On the right (3) the cone was on $4 \mathrm{~mm}$ inside the outlet of nozzle.

from dynamic emitter radiate in wide range. A case of infrared radiation is shown on the right of Figure 3. Maximal intensity is detected in the nodes of the structure. The flow temperature was $285^{\circ} \mathrm{K}$ measured with probe temperature sensor, and pre-chamber pressure was 4 - $6 \mathrm{~atm}$. The radiation was registered with monochromator of diffractive-grating type. Thus the motion of the jet is accompanied by the radiation within the ultraviolet, visible and infrared spectrum [6,7]. The dark spots on the boundary of Mach shock waves on the right picture (3) indicate the infrared radiation emission.

\subsection{Thermal Field of Radiating Plasma}

To elucidate character of super-compressibility of supersonic spiral-twisted air jets during their flowing from the nozzle with a central cone we performed the second experiment. The infrared radiation of spiral-twisted air jets during interacting with plastic plate was studied. We have to need to ensure that energy radiation cool effectively the plate due to internal gas energy decrease, kinetic gas energy increase and emission of infrared radiation.

Here in Figure 4 the pressure in pre-chamber was 4 - 6 atm, the temperature of spiral-twisted air jets was $285^{\circ} \mathrm{K}$. The environment room temperature was $293^{\circ} \mathrm{K}$. The air jet was directed on the plate with size $10 \times 10 \mathrm{~cm}^{2}$ and thickness $0.5 \mathrm{~cm}$ disposed perpendicular to the direction of the jet on distance $3 \mathrm{~cm}$ from protruding part of the central cone.

The thermal field of radiating plasma on reverse side of the plastic plate was measured. The thermal field of radiating plasma on reverse side of the plastic plate is presented on the left of Figure 4. The diagram of thermal the field of radiating plasma is presented on the right of Figure 4. The degrees are everywhere in ${ }^{\circ} \mathrm{C}$.

The delamination of flow in Figure $\mathbf{4}$ on coaxial type channels with different temperatures is observed. One can see "deer foot print" on the side that is opposite to the side interacting with the jet. It means that strong interaction occurs between jet and plate material that is transferred on long distances without energetic losses and without disruption of the structure of jet.

\subsection{Radiation of High Energy Detecting in Water Vapor Subsonic and Supersonic Jets}

Taking in account our results with air flow we decided to use in the dynamic emitter water vapor for increasing the energy density. During the flowing of the vapor in the atmosphere the characteristics of plasma with more density of energy were measured. The emission of electromagnetic radiation from the X-ray to infrared spectrum has been found from initially neutral vapor. The results see below in Table 1:

The accuracy of measurements was $0.5 \%$ at the multiple repetitions of the experiments.

In the third experiment with the same nozzle was supplied water vapor. The pressure of water vapor in prechamber was 2 - 12 atm (as in Table 1), the temperature of vapor was $393^{\circ} \mathrm{K}$ in the pre-chamber and it decreased to $333^{\circ} \mathrm{K}$ on output of the dynamic emitter. The environment room temperature was $293^{\circ} \mathrm{K}$.

Beginning from 4 atm pressure, the high frequency field was detected from $1.4 \mathrm{MHz}$ up to $1 \mathrm{GHz}$ in spiral-twisted supersonic jets. The field arises in region both in and around of the jet.

In Figures $\mathbf{2}$ and $\mathbf{3}$ one can see that the radiation in submerged jet has a regular character due to regularly interacting shock waves [7]. Therewith in Figure 2 one can see glowing space around the jet. In case of supplying water vapor supersonic jets start glowing in violet region and other in region of other short electromagnetic waves. Glowing in violet region is visually observed with the naked eye in dark conditions on approaching a conducting mass by $1-2 \mathrm{~m}$.

The increased brightness of discrete parts of the jet appears as a result of high electrons density. Water vapor 

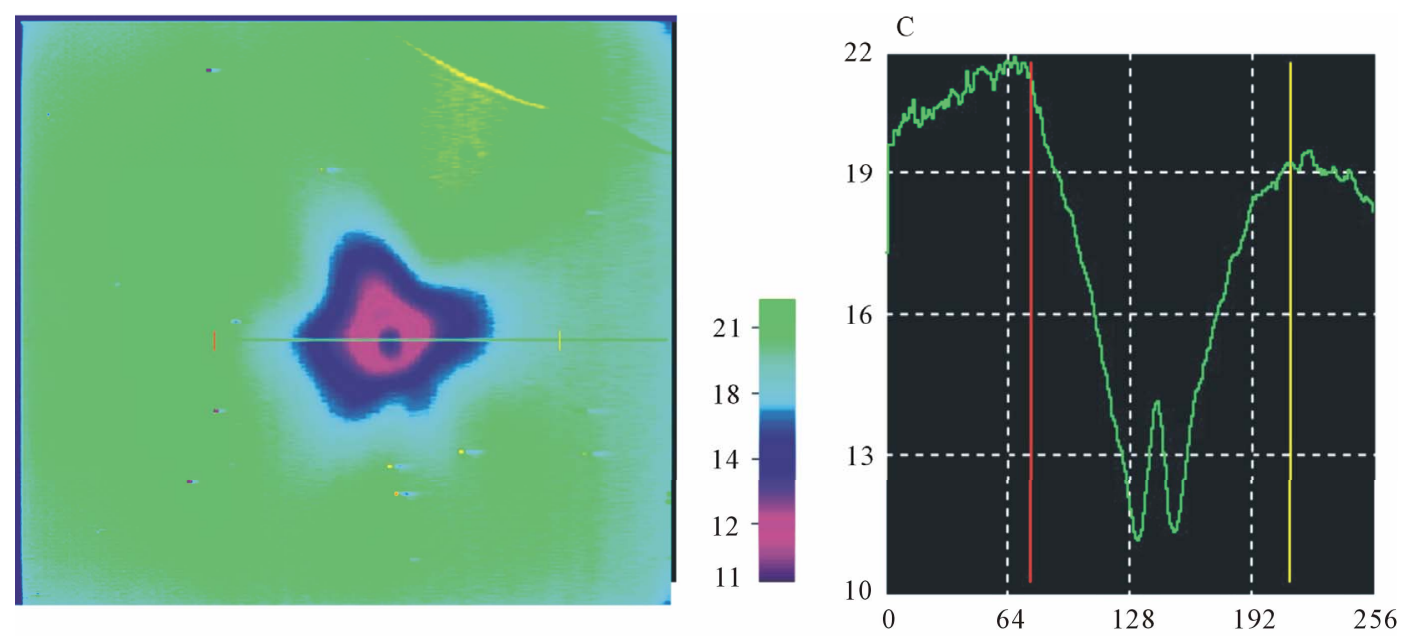

Figure 4. Thermal field (in ${ }^{\circ} \mathrm{C}$ ) of radiating plasma measured on reverse side of polistirol plastic plate. "Deer foot print" on the left demonstrates the layered structure in cross-section of the jet.

Table 1. Electromagnetic characteristics of spiral twisted vapor-air jet.

\begin{tabular}{|c|c|c|c|}
\hline No. & Electromagnetic characteristics of vapor-air plasma & Value & Note \\
\hline 1 & $\begin{array}{l}\text { High frequency voltage of the jet for various } \\
\text { pressures and emitters }\end{array}$ & $\begin{array}{c}3-4 \mathrm{kV} \\
50-80 \mathrm{kV}\end{array}$ & $\begin{array}{l}\text { pre-chamber pressure } 2-4 \mathrm{~atm} ; 393^{\circ} \mathrm{K} \\
\text { pre-chamber pressure } 10-12 \mathrm{~atm} ; 393^{\circ} \mathrm{K}\end{array}$ \\
\hline 2 & $\begin{array}{l}\text { Frequency of electric field for various pressures and } \\
\text { emitters }\end{array}$ & $1.4 \mathrm{MHz}-1 \mathrm{GHz}$ & pre-chamber pressure $4-8 \mathrm{~atm}$ \\
\hline 3 & $\begin{array}{l}\text { Direct current voltage of the jet for various pressures } \\
\text { and emitters }\end{array}$ & $80-100 \mathrm{kV}$ & pre-chamber pressure $10-12 \mathrm{~atm} ; 393^{\circ} \mathrm{K}$ \\
\hline 4 & Electric field strength around of the jet & $10^{2} \mathrm{~V} / \mathrm{cm}$ & at distance $r=5-10 \mathrm{~m}$ \\
\hline 5 & $\begin{array}{l}\text { Amplitude electric current for HF field for various } \\
\text { pressures and emitters }\end{array}$ & $1 \mu \mathrm{A}-1 \mathrm{~mA}$ & $\begin{array}{l}\text { during short-circuit of the jet on the } \\
\text { ground; at pressures } 6-12 \text { atm }\end{array}$ \\
\hline 6 & Electrons density in different layers of the jet & $10^{9} \mathrm{~cm}^{-3}$ & at distance $\mathrm{r}=1 \mathrm{~m}$ in vapor-air jet \\
\hline 7 & Electrons density near the central cone & $10^{15} \mathrm{~cm}^{-3}$ & at distance $\mathrm{r}=10 \mathrm{~mm}-10 \mathrm{~cm}$ from cone \\
\hline
\end{tabular}

provides plasma with more specific density of electrons energy [3] that provides energy cumulation in small volumes and high energy radiation.

One can see that measured characteristics are enough for generation of radiation of high energy as result of super-compressibility of internal energy decreasing and kinetic energy increasing of supersonic spiral-twisted jets in the vapor-air structured flows.

\subsection{Study of Spectral Characteristics during Super-Compressibility of Supersonic Jets}

We had reported about emission of electromagnetic radiation from the X-ray to infrared spectrum from initially neutral water vapor jets flowing from the nozzle with a central cone [4].

To provide study of radiation characteristics during super-compressibility of supersonic jets, corona dis- charge was used as the mean for the jet visualization and its spectra registration.

Corona discharge is an electrical discharge that occurs when the strength or potential gradient of the electrical field around the conductor is high enough to form a conductive region, but not high enough to cause electrical breakdown or arcing to nearby objects. In our case the conductor is central cone in pre-chamber (1 in Figure 1).

The overview spectra of emission of radiation of the jets were obtained in region $\lambda=280-800 \mathrm{~nm}, \Delta \lambda=$ $0.293 \mathrm{~nm}$ with AVASPEC 2048. Detailed spectra of emission of radiation were obtained in optical region $\lambda=$ $383-527 \mathrm{~nm}, \Delta \lambda=0.083 \mathrm{~nm}$.

The visualization was provided with electric discharge from $\mathrm{HF}$ generator, where frequency was $f=268 \mathrm{kHz}$, output inductivity was $L=21 \mathrm{mHn}$, generator power was $700-800 \mathrm{~W}$, current amplitude $I_{A}=0.5 \mathrm{~A}$, voltage am- 
plitude $U_{A}=5 \mathrm{kV}$.

In Figure 5 corona discharge starting from the top of cone is presented.

The decoding of the spectra in Figure 6 as Figures 7 and $\mathbf{8}$ allowed us to define the inverted population of vibrational levels for nitrogen molecular ions $\left(\mathrm{N}_{2}^{+}\right)$. The decoding of the spectra in Figure 9 allowed us estimating electrons temperature $\mathrm{T}_{\mathrm{e}}=5000^{\circ} \mathrm{K}$, rotational temperature for nitrogen molecular ions $\left(\mathrm{N}_{2}^{+}\right) \quad \mathrm{T}_{\mathrm{rN}}=$ $2900^{\circ} \mathrm{K}$ and vibrational temperature for nitrogen molecular ions $\left(\mathrm{N}_{2}^{+}\right) \mathrm{T}_{\mathrm{vN}}=5000^{\circ} \mathrm{K}$. Electron density was estimated from broadening of hydrogen lines equal from $10^{14}$ to $10^{16} \mathrm{~cm}^{-3}$.

These results are in good agreement with measurements and estimations of plasma characteristics of spiral twisted vapor-air jets flowing from the dynamic emitter with central cone (see Table 1).

\section{Conclusions}

1) New source of light emission of high energy is found due to arising of instability in supersonic jets. High energy density is detected in gas flowing from the nozzle with a central cone.

2) The super-compressibility is observed in supersonic jets as result of gas adiabatic expansion and its internal energy decreasing. It is provided due to internal forces and as a result of intensive interacting of the accelerated molecules in the gas and jets structures. The internal forces lead to super-compressibility of the jets and to its volume decreasing.

3) Internal energy decreasing generates the instability; super-compressibility is result of gas volume decreasing; its produce high density of light energy emission in continuous media.

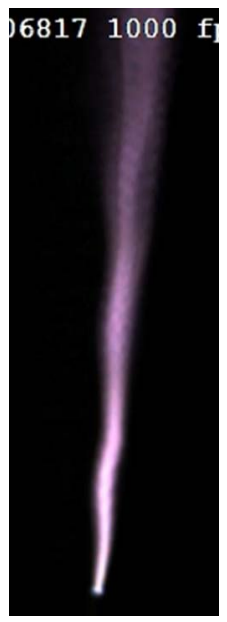

Figure 5. Corona discharge starting from top of the central cone of dynamic emitter. One can see spiral structure in the violet luminous part of the jet. Pre-chamber pressure was $\mathbf{8} \cdot \mathbf{a t m}$. Here shutter speed was $\mathbf{1 0 0 0}$ frames per seconds. Corona discharge was provided to obtain the spectra.

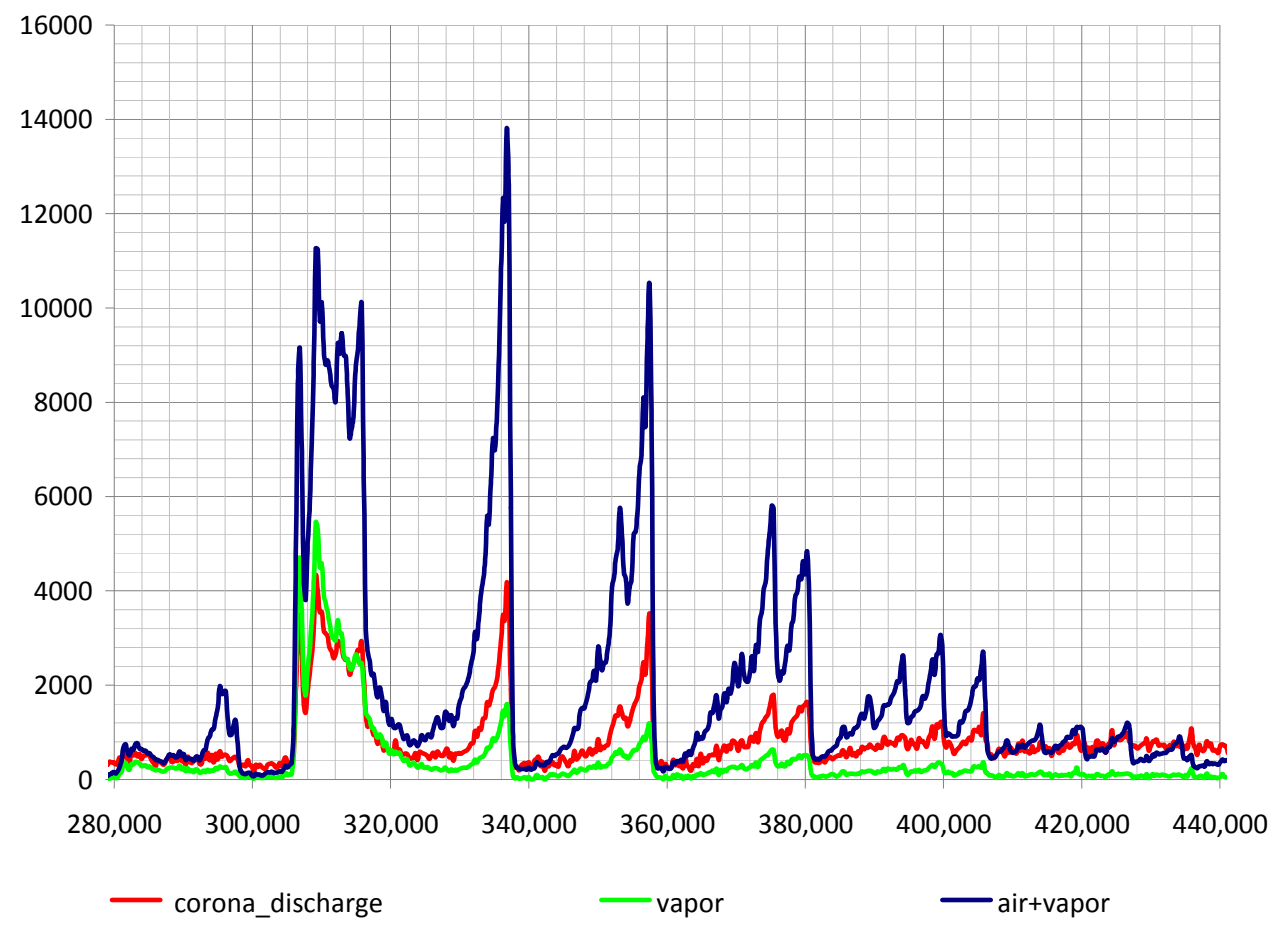

Figure 6. The emission spectrum of the jets. Three cases of emission correspond to the three curves. Red curve presents external corona discharge without jets. Green curve presents jet of vapor in corona. Dark blue curve jet presents air jet with vapor in corona discharge. Last case characterized with more concentration of ions and is more intensive. The temperature for electrons is $\mathbf{T}_{e}=5_{000}{ }^{\circ} \mathrm{K}$ for case of dark blue curve. 


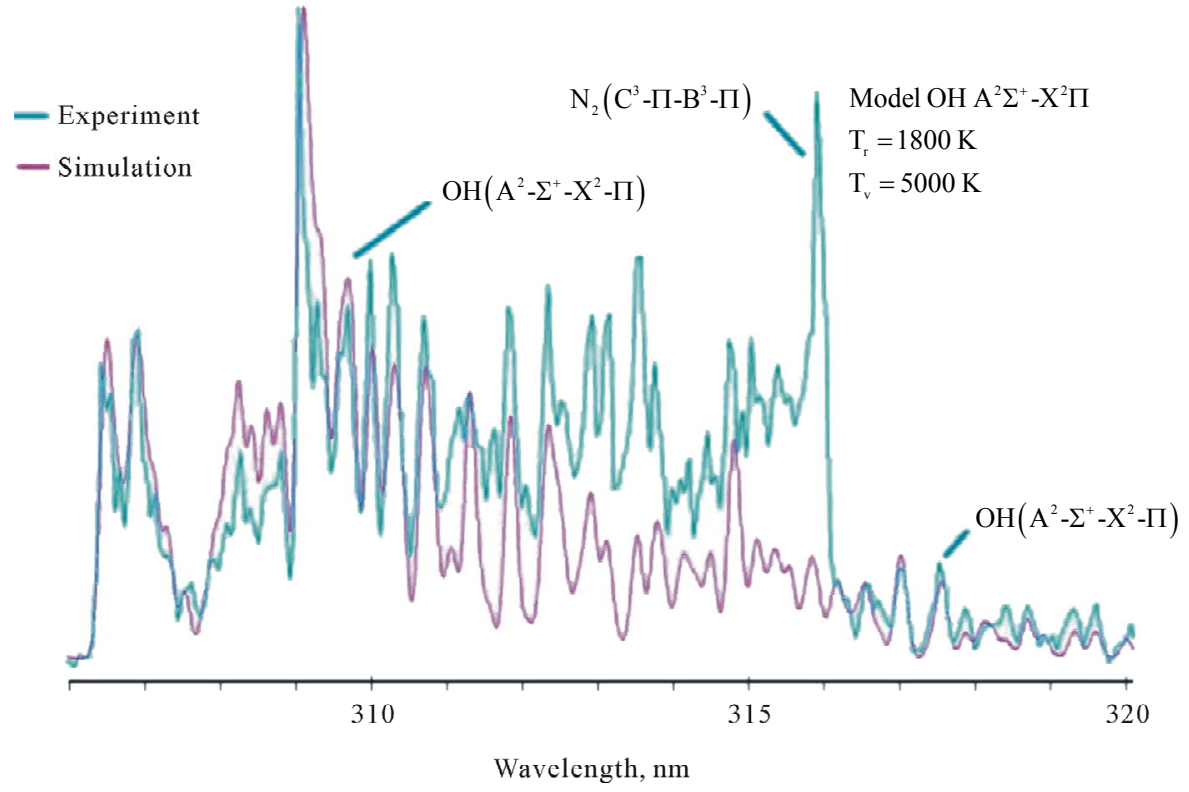

Figure 7. Emission spectrum of the jet with the discharge. Violet curve corresponds to the model of emission of $\left(\mathrm{OH}^{-}\right)$. $\mathrm{Blue}^{-}$ curve corresponds to the experimental curve of emission of $\left(\mathrm{OH}^{-}\right)$and $\left(\mathrm{N}_{2}^{+}\right)$ions. Here the rotational temperature for $\left(\mathrm{OH}^{-}\right)$ ions is equal $T_{r}=1800^{\circ} \mathrm{K}$ and vibrational temperature for $\left(\mathrm{OH}^{\circ}\right)$ ions is equal $\mathrm{T}_{v}=5000^{\circ} \mathrm{K}$.

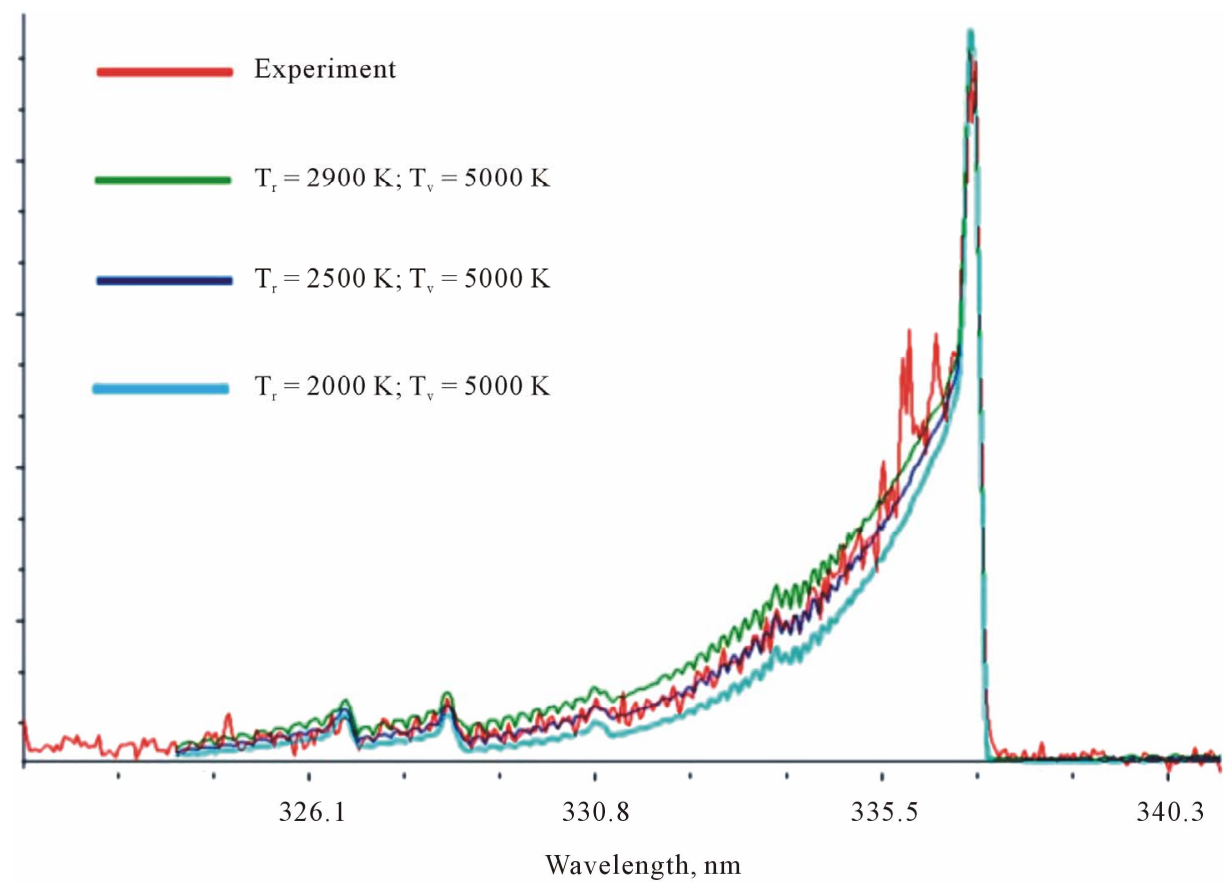

Figure 8. Decoded detailed emission spectra for the jets from Figure 6. The four cases of emission correspond to four curves in blue-violet region of light emission: Red (EXP) curve presents experimental results of emission of the discharge without the jet. Green curve presents model of rotational temperature for ions $\left(\mathrm{N}_{2}^{+}\right)$in presence of vapor with high pressure, here air rotational temperature in the jet $T_{r}=2900^{\circ} \mathrm{K}$ and vibrational temperature for ions $\left(N_{2}^{+}\right)$is $T_{v}=5000^{\circ} \mathrm{K}$. Dark blue curve presents model of rotational temperature for ions $\left(\mathrm{N}_{2}^{+}\right)$in the jet without vapor with high pressure, here air rotational temperature in the jet $T_{r}=2500^{\circ} \mathrm{K}$ and vibrational temperature for ions $\left(N_{2}^{+}\right)$is $T_{v}=5000^{\circ} \mathrm{K}$. Blue curve presents model of rotational temperature for ions $\left(\mathrm{N}_{2}^{+}\right)$in weak (less than 1 barr) vapor with low pressure, here air rotational temperature in the jet $T_{r}=2000$ and vibrational temperature for ions $\left(N_{2}^{+}\right)$is $T_{v}=5000^{\circ} \mathrm{K}$. 


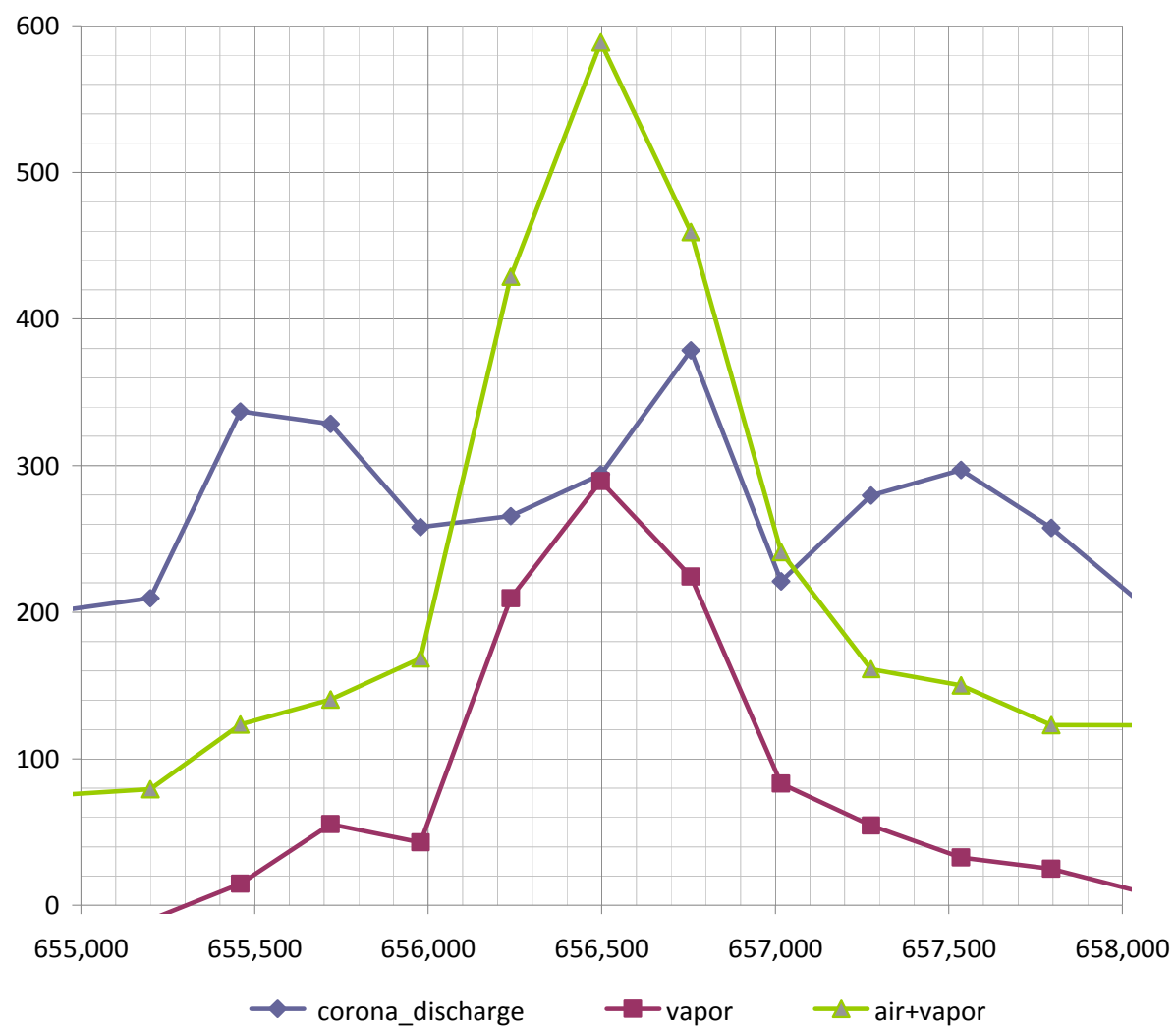

Figure 9. Detailed spectrum from Figure 6 near spectrum line $656.5 \mathrm{~nm}$ of $\mathrm{H}_{\alpha}$ hydrogen. Three cases of emission correspond to the three curves: external corona discharge without jet (blue curve), jet of vapor in corona (red curve) and jet of air with vapor in corona discharge (green curve).

\section{Acknowledgements}

Author deeply appreciate to: B. V. Melkoumian from A. M. Prokhorov General Physics Institute of Russian Academy of Sciences for comments, discussions and help in paper preparation; A. I. Klimov, A. S. Paschina and A. Efimov from Jointed Institute of High Temperature of Russian Academy of Sciences for obtaining of the emission spectra of the jets.

Author gratefully acknowledges for the long-term support and consulting to M. V. Lomonosov Moscow State University Rector, academician V. A. Sadovnichiy.

\section{REFERENCES}

[1] Kh. Khasanov, "Visualization of Super-Compressibility in Supersonic Spiral-Twisted Jets," Physics Letters A, Vol. 376, No. 5, 2012, pp. 748-753. doi:10.1016/j.physleta.2011.12.018

[2] Kh. Khasanov, "Bi-Spiral Twisted Supersonic Gas Flow. News of Russian Academy of Sciences," Mechanics of Fluids and Gases, No. 3, 2011, pp. 106-110.

[3] Kh. Khasanov, "Visualization of Bi-Spiral Twisted Supersonic Gas Flow," The 8th Pacific Symposium of Flow Visualization and Image Processing, Moscow, 21-25 August 2011, p. 51.
[4] Kh. Khasanov, "Quantum Antigravitation of Vapor-Air Jet," International Symposium on Molecular Spectroscopy, 56th Meeting, Abstracts, Ohio State University, Columbus, 11-15 June 2001, p. 199.

[5] Kh. Khasanov and S. V. Petukhov, "Dynamic Emitter," RF Patent No. 2058196, 1996.

[6] Kh. Khasanov, "Bi-Spiral Switched Supersonic Jet Flow Escaping from a Circular Nozzle: Interaction with Metal and Polymer Screens, Infrared Radiation Phenomenon," International Conference: Fluxes and Structures in Fluids. Physics of Geospheres-2009, Moscow, 24-27 June 2010, pp. 206- 210.

[7] Kh. Khasanov, "Radiation of Bi-Spiral Switched Supersonic Jet Flow Escaping from an Annular Nozzle," Fluxes and Structures in Fluids. Physics of Geospheres-2009, Abstracts, Moscow, 2010.

[8] W. B. McNamara, Y. T. Didenko and K. S. Suslick, "Sonoluminescence Temperatures during Multi-Bubble Cavitation, Nature, Vol. 401, No. 6755, 1999, pp. 772775.

[9] K. Mohseni, T. Colonius and J. B. Freund, "An Evaluation of Linear Instability Waves as Sources of Sound in a Supersonic Turbulent Jet," Physics of Fluids, Vol. 14, No. 10, 2002, pp. 3593-3600. doi:10.1063/1.1501545

[10] P. Hartigan, A. Frank, J. M. Foster, B. H. Wilde, M. Douglas, P. A. Rosen, R. F. Coker, B. E. Blue and J. F. Hansen, "Fluid Dynamics of Stellar Jets in Real Time: 
Third Epoch Hubble Space Telescope Images of HH 1, HH 34, And HH 47,", Astrophysical Journal, Vol. 736, No. 1, 2011, p. 29. doi:10.1088/0004-637X/736/1/29

[11] D. W. Savin, et al., "The Impact of Recent Advances in Laboratory Astrophysics on Our Understanding of the Cosmos," Reports on Progress in Physics, Vol. 75, No. 3,
2012, pp. 1-36.doi:10.1088/0034-4885/75/3/036901

[12] D. Tordella, M. Belan, S. Massaglia, S. De Ponte, A. Mignone, E. Bodenschatz and A. Ferrari, "Astrophysical Jets: Insights into Long-Term Hydrodynamics," New Journal of Physics, Vol. 13, No. 4, 2011, Article ID: 043011. doi:10.1088/1367-2630/13/4/043011 